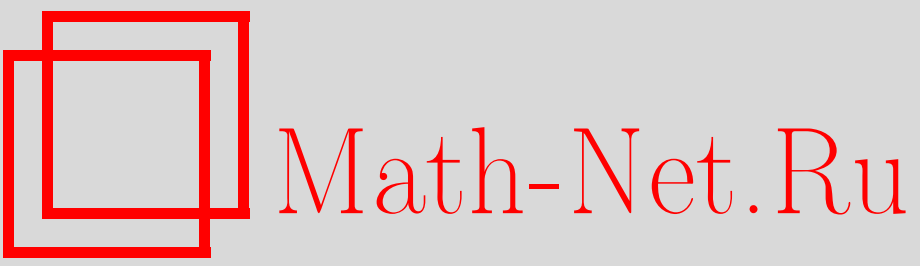

H.

A.

Раутиан,

Корректная разрешимость вольтерровых интегро-дифференциальных уравнений с сингулярными ядрами, Итоги науки и техн. Сер. Соврем. мат. и ее прил. Темат. обз., 2021, том 191, 135-148

DOI: https://doi.org/10.36535/0233-6723-2021-191-135-148

Использование Общероссийского математического портала Math-Net.Ru подразумевает, что вы прочитали и согласны с пользовательским соглашением

http://www.mathnet.ru/rus/agreement

Параметры загрузки:

IP : 52.90 .164 .192

26 апреля 2023 г., 05:11:58 
ИТОГИ НАУКИ И ТЕХНИКИ.

Современная математика и ее приложения.

Тематические обзоры.

Том 191 (2021). С. 135-148

DOI: 10.36535/0233-6723-2021-191-135-148

УДК 517.968.72

\title{
КОРРЕКТНАЯ РАЗРЕШИМОСТЬ ВОЛЬТЕРРОВЫХ ИНТЕГРО-ДИФФЕРЕНЦИАЛЬНЫХ УРАВНЕНИЙ \\ С СИНГУЛЯРНЫМИ ЯДРАМИ
}

\author{
(c) 2021 г. H. А. РАУТИАН
}

\begin{abstract}
АннотАция. Работа посвящена изучению интегро-дифференциальных уравнений с неограниченными операторными коэффициентами в гильбертовом пространстве. Главная часть рассматриваемых уравнений представляет собой абстрактное гиперболическое уравнение, возмущенное слагаемыми, содержащими вольтерровы интегральные операторы с сингулярными ядрами. Указанные уравнения представляют собой абстрактную форму интегро-дифференциального линейной вязкоупругости, уравнения Гуртина-Пипкина, описывающего процесс распространения тепла в средах с памятью, а также интегро-дифференциальных уравнений, возникающих в задачах усреднения в перфорированных средах (закон Дарси). Устанавливается условия существования и единственности сильных и обобщенных решений начально-краевых задач для указанных уравнений в весовых пространствах Соболева на положительной полуоси.
\end{abstract}

Ключевые слова: интегро-дифференциальное уравнение, оператор-функция, корректная разрешимость

\section{WELL-POSEDNESS OF VOLTERRA INTEGRO-DIFFERENTIAL EQUATIONS WITH SINGULAR KERNELS}

\author{
(c) $2021 \quad$ N. A. RAUTIAN
}

\begin{abstract}
This paper is devoted to the study of integro-differential equations with unbounded operator coefficients in a Hilbert space. The principal part of the equations considered is an abstract hyperbolic equation perturbed by terms containing Volterra integral operators with singular kernels. Such integro-differential equations describe, for example, linear viscoelastic phenomena, heat propagation processes in media with memory (the Gurtin-Pipkin equation), averaging problems in perforated media (Darcy's law), etc. We establish conditions for the existence and uniqueness of strong and generalized solutions to initial-boundary-value problems for these equations in weighted Sobolev spaces on the positive semiaxis.
\end{abstract}

Keywords and phrases: integro-differential equation, operator function, well-posedness.

AMS Subject Classification: 47G20, 34K30, 47A56, 34K12

1. Введение. Рассмотрим простейший пример рассматриваемых в статье уравнений, которым является одномерное уравнение линейной вязкоупругости

$$
u_{t t}(x, t)=u_{x x}(x, t)-\int_{0}^{t} K(t-\tau) u_{x x}(x, \tau) d \tau+f(x, t),
$$

Работа выполнена при поддержке Российского фонда фундаментальных исследований (проект № 20-01-00288а). 
где функция ядра $K(t)$ является неотрицательной, монотонно убывающей функцией при $t>0$ и называется ядром релаксации. Выбор ядра релаксации определяется характеристиками рассматриваемого материала.

Математическая модель вязкоупругости описывает поведение материалов, механические свойства которых зависят не только от деформации в данный момент времени, а учитывают всю историю деформирования материала (т.е. линейные определяющие уравнения более общего вида). Такие материалы в литературе называются материалами с памятью (см. $[17,18])$. В настоящее время существует обширная литература, посвященная изучению материалов с памятью, как прикладного, так и фундаментального характера, посвященная построению математических моделей и развитию математических методов исследования указанных материалов $($ см. $[4,11-14,19]$ и. т. д.). Необходимость в изучении вязкоупругих материалов появилась еще в 50-е годы прошлого века, в связи с развитием химической промышленности и появлением различных полимерных и композитных материалов. В настоящее время большой интерес представляет направление исследований, связанное с изучением биополимеров, моделирующих поведение биологических тканей. Например, в [25, 27] изучаются вязкоупругие свойства синтетических тканей, обладающих качественными характеристиками костей человека, а также тканей искусственного сердца. В $[22,28]$ приведены модели вязкоупругости, нашедшие применение в пищевой промышленности.

Анализ уравнения (1) можно было бы существенно упростить, предположив, что ядро релаксации имеет вид

$$
\sum_{j=1}^{N} c_{j} e^{-\gamma_{j} t}, \quad t>0,
$$

где и $\gamma_{j}$ - некоторые положительные постоянные, поскольку в этом случае уравнение (1) нетрудно свести к дифференциальному (см. [20]). Однако при обработке экспериментальных данных оказалось, что ядра вида (2) плохо подходят для описания поведения реальных материалов. Для того чтобы учесть наблюдаемые в экспериментах закономерности, необходимо использовать сингулярные ядра релаксации, т.е. ядра, стремящиеся к бесконечности при $t \rightarrow+0$. Еще Больщман предлагал выбрать сингулярное ядро релаксации в виде

$$
K(t)=\frac{k}{t}, \quad t>0,
$$

где $k>0$. Однако ядро (3) обладает неинтегрируемой особенностью при $t \rightarrow+0$, и использовать его в уравнении (1) невозможно.

Простейшим сингулярным ядром релаксации, пригодным для использования в линейной наследственной модели, является так называемое ядро Абеля

$$
K(t)=\frac{k}{t^{\alpha}}, \quad t>0,
$$

где $k>0,0<\alpha<1$. Следует заметить однако, что для широкого класса материалов в испытаниях на релаксацию напряжение падает на больших временах быстрее, чем это может обеспечить ядро вида (4).

Стремление получить ядра, обладающие той же сингулярность, что и ядро Абеля, и достаточно быстро убывающие при $t \rightarrow+0$, привело к построению целого набора ядер (см. [26]). Большое распространение получило ядро Работнова - дробно-экспоненциальная функция (см. [13]).

В нашей работе рассматривается абстрактное ядро релаксации вида,

$$
K(t)=\int_{0}^{+\infty} e^{-t \tau} d \mu(\tau),
$$

которое представляет собой преобразование Лапласа положительной меры $d \mu$ и объединяет широкий класс сингулярных ядер, возникающих в приложениях.

Следует также отметить, что уравнение вида (1) носит название уравнения Гуртина-Пипкина и описывает процесс распространения тепла в средах с памятью с конечной скоростью, а также возникает в задачах усреднения в перфорированных средах (закон Дарси). 
В нашей работе мы получаем достаточные условия существования и единственности сильных и обобщенных решений начальных задач для абстрактных интегро-дифференциальных уравнений в весовых пространствах Соболева вектор-функций на положительной полуоси со значениями в гильбертовом пространстве. Указанные задачи могут быть реализованы как начально-краевые задачи для интегро-дифференциальных уравнений с частными производными в областях с достаточно гладкой границей для уравнений Гуртина-Пипкина в весовых пространствах Соболева на положительной полуоси и исследуем некоторые спектральные вопросы для оператор-функций, являющихся символами указанных уравнений.

Предлагаемая работа является естественным продолжением исследований, начатых в [2-9].

\section{2. Определения, обозначения, формулировка результатов.}

2.1. Определения и обозначения. Пусть $H$-сепарабельное гильбертово пространство, $A$ - самосопряженный положительный оператор, $A^{*}=A$, действующий в пространстве $H$, имеющий компактный обратный.

$$
\begin{gathered}
\frac{d^{2} u}{d t^{2}}+A^{2} u-\int_{0}^{t} K(t-s) A^{2} u(s) d s=f(t), \quad t \in \mathbb{R}_{+}, \\
u(+0)=\varphi_{0}, \\
u^{(1)}(+0)=\varphi_{1} .
\end{gathered}
$$

Скалярная функция $K(t)$ имеет следующее представление:

$$
K(t)=\int_{0}^{\infty} e^{-t \tau} d \mu(\tau),
$$

где $d \mu$ - положительная мера, которой соответствует возрастающая, непрерывная справа функция распределения $\mu$. Интеграл понимается в смысле Стильтьеса. Мы предполагаем, что выполнено следующее условие:

$$
\int_{0}^{\infty} \frac{d \mu(\tau)}{\tau}<1
$$

где носитель $\mu$ принадлежит полуоси $(0,+\infty)$. Из условия $(10)$ следует, что функция $K(t)$ принадлежит пространству $L_{1}\left(\mathbb{R}_{+}\right)$.

В качестве примера можно рассмотреть функцию $K(t)$ вида

$$
K(t)=\sum_{j=1}^{\infty} c_{j} R_{j}(t)
$$

где $c_{j}>0, j \in \mathbb{N}$, функции $R_{j}(t)$ - дробно-экспоненциальные функции вида (см. [13, гл. І])

$$
R_{j}(t)=t^{\alpha-1} \sum_{n=0}^{\infty} \frac{\left(-\beta_{j}\right)^{n} t^{n \alpha}}{\Gamma[(n+1) \alpha]}, \quad 0<\alpha<1,
$$

$\Gamma(\cdot)$ - гамма функция Эйлера. При этом предполагается, что последовательность $\left\{\beta_{j}\right\}$ удовлетворяет условиям $0<\beta_{j}<\beta_{j+1}, j \in \mathbb{N}, \beta_{j} \rightarrow+\infty, j \rightarrow+\infty$. Кроме того, выполнены условия

$$
\sum_{j=1}^{\infty} \frac{c_{j}}{\beta_{j}}<1 \text {. }
$$

Преобразование Лапласа функции $R_{j}(t)$ имеет вид

$$
\hat{R}_{j}(\lambda)=\frac{1}{\lambda^{\alpha}+\beta_{j}}
$$


(см. [13, гл. I]). При этом под $\lambda^{\alpha}(0<\alpha<1)$ понимается главная ветвь многозначной функции $f(\lambda)=\lambda^{\alpha}, \lambda \in \mathbb{C}$ с разрезом по отрицательной действительной полуоси $\lambda^{\alpha}=\left|\lambda^{\alpha}\right| e^{i \alpha \arg \lambda}$, $-\pi<\arg \lambda<\pi$. При помощи обратного преобразования Лапласа, примененного к главной ветви функции $\hat{R}_{j}(\lambda)$, получено (см. [13, гл. I]) следующее интегральное представление функции $R_{j}(t)$ :

$$
R_{j}(t)=\frac{1}{2 \pi i} \lim _{R \rightarrow+\infty} \int_{\gamma-i R}^{\gamma+i R} \frac{e^{\lambda t} d \lambda}{\lambda^{\alpha}+\beta_{j}}=\frac{\sin \pi \alpha}{\pi} \int_{0}^{+\infty} \frac{e^{-t \tau} d \tau}{\tau^{\alpha}+2 \beta_{j} \cos \pi \alpha+\beta_{j}^{2} \tau^{-\alpha}} .
$$

Рассматривая преобразование Лапласа уравнения (6) при однородных начальных условиях, получаем уравнение $L(\lambda) \hat{u}(\lambda)=\hat{f}(\lambda)$, где оператор-функция

$$
L(\lambda)=\lambda^{2} I+A^{2}-\hat{K}(\lambda) A^{2}
$$

является символом этого уравнения, а $\hat{u}(\lambda)$ и $\hat{f}(\lambda)$ - преобразования Лапласа вектор-функций $u(t)$ и $f(t)$, соответственно. Здесь $\hat{K}(\lambda)$ - преобразование Лапласа ядра $K(t)$, имеющие представление

$$
\hat{K}(\lambda)=\int_{0}^{\infty} \frac{d \mu(\tau)}{\lambda+\tau}
$$

Через $W_{2, \gamma}^{n}\left(\mathbb{R}_{+}, A^{n}\right)$ обозначим пространство Соболева вектор-функций на полуоси $\mathbb{R}_{+}=(0, \infty)$ со значениями в $H$, снабженное нормой

$$
\|u\|_{W_{2, \gamma}^{n}\left(\mathbb{R}_{+}, A^{n}\right)} \equiv\left(\int_{0}^{\infty} e^{-2 \gamma t}\left(\left\|u^{(n)}(t)\right\|_{H}^{2}+\left\|A^{n} u(t)\right\|_{H}^{2}\right) d t\right)^{1 / 2}, \quad \gamma \geqslant 0 .
$$

Подробнее о пространствах $W_{2, \gamma}^{n}\left(\mathbb{R}_{+}, A^{2}\right)$ см. монографию [21, глава 1]. Для $n=0$ полагаем $W_{2, \gamma}^{0}\left(\mathbb{R}_{+}, A^{0}\right)=L_{2, \gamma}\left(\mathbb{R}_{+}, H\right)$, где $L_{2, \gamma}\left(\mathbb{R}_{+}, H\right)$ обозначено пространство измеримых функций со значениями в пространстве $H$, снабженное нормой

$$
\|f\|_{L_{2, \gamma}\left(\mathbb{R}_{+}, H\right)}=\left(\int_{0}^{+\infty} e^{-2 \gamma t}\|f(t)\|_{H}^{2} d t\right)^{1 / 2} .
$$

Определение 1. Будем называть вектор-функцию $u$ сильным решением задачи (6)-(8), если она принадлежит пространству $W_{2, \gamma}^{2}\left(\mathbb{R}_{+}, A^{2}\right)$ для некоторого $\gamma \geqslant 0$, удовлетворяет уравнению (6) почти всюду на полуоси $\mathbb{R}_{+}$, и начальным условиям $(7),(8)$.

Определение 2. Будем называть вектор-функцию $u$ обобщенным решением задачи (6)-(8), если она принадлежит пространству $W_{2, \gamma}^{1}\left(\mathbb{R}_{+}, A\right)$ для некоторого $\gamma \geqslant 0$, удовлетворяет начальному условию (7) и удовлетворяет тождеству

$$
\begin{aligned}
\left\langle A\left[u(t)-\int_{0}^{t} K(t-s) u(s) d s\right], A v(t)\right\rangle_{L_{2, \gamma}\left(\mathbb{R}_{+}, H\right)}-\left\langle u^{\prime}(t), v^{\prime}(t)\right\rangle_{L_{2, \gamma}\left(\mathbb{R}_{+}, H\right)}+ \\
+2 \gamma\left\langle u^{\prime}(t), v(t)\right\rangle_{L_{2, \gamma}\left(\mathbb{R}_{+}, H\right)}=\langle f(t), v(t)\rangle_{L_{2, \gamma}\left(\mathbb{R}_{+}, H\right)}+\left(\varphi_{1}, v(0)\right)_{H}
\end{aligned}
$$

при всех $v(t) \in W_{2, \gamma}^{1}\left(\mathbb{R}_{+}, A\right)$, удовлетворяющих условию

$$
\lim _{t \rightarrow+\infty} v(t) e^{-2 \gamma t}=0 .
$$

Превратим область определения $\operatorname{Dom}\left(A^{\beta}\right)$ оператора $A^{\beta}, \beta>0$ в гильбертово пространство $H_{\beta}$, введя на $\operatorname{Dom}\left(A^{\beta}\right)$ норму $\|\cdot\|_{\beta}=\left\|A^{\beta} \cdot\right\|$, эквивалентную норме графика оператора $A^{\beta}$. 


\section{2. Формулировка результатов.}

Теорема 1. Предположим, что вектор-функция $A f(t) \in L_{2, \gamma_{0}}\left(\mathbb{R}_{+}, H\right)$ для некоторого $\gamma_{0}>0$, ядро $K(t)$ представимо в виде (9), а такэе выполняется условие (10), кроме того, $\varphi_{0} \in H_{3}$, $\varphi_{1} \in H_{2}$. Тогда существует такое $\gamma_{1}>\gamma_{0}$, что для всех $\gamma \geqslant \gamma_{1}$ задача (6)-(8) имеет единственное сильное решение в пространстве $W_{2, \gamma}^{2}\left(\mathbb{R}_{+}, A^{2}\right)$, удовлетворяющее неравенству

$$
\|u\|_{W_{2, \gamma}^{2}\left(\mathbb{R}_{+}, A^{2}\right)} \leqslant d\left(\|A f\|_{L_{2, \gamma}\left(\mathbb{R}_{+}, H\right)}+\left\|A^{3} \varphi_{0}\right\|_{H}+\left\|A^{2} \varphi_{1}\right\|_{H}\right),
$$

с постоянной $d$, не зависящей от вектор-функции $f$ и векторов $\varphi_{0}, \varphi_{1}$.

Следует отметить, что метод, используемый нами для доказательства корректной разрешимости начальных задач для абстрактных интегро-дифференциальных уравнений, существенно отличается от более традиционного подхода, использованного Л. Пандолфи в [23], где разрешимость изучается в функциональных пространствах на конечном временном интервале $(0, T)$. В нашей работе разрешимость изучается в весовых пространствах Соболева $W_{2, \gamma}^{2}\left(\mathbb{R}_{+}, A\right)$ векторфункций на положительной полуоси $\mathbb{R}_{+}$, где $A$ - положительный самосопряженный оператор в гильбертовом пространстве. Доказательство нашей теоремы 1 о разрешимости существенно использует гильбертову структуру пространств $W_{2, \gamma}^{2}\left(\mathbb{R}_{+}, A\right), L_{2, \gamma}\left(\mathbb{R}_{+}, H\right)$, а также теорему ПэлиВинера.

В настоящее время имеется обширная литература, посвященная изучению абстрактных интегро-дифференциальных уравнений, т.е. уравнений с неограниченными операторными коэффициентами в банаховых и гильбертовых пространствах. Ограничимся здесь указанием работ [14-20,22-31] (см. также указанную там библиографию).

Исследованию интегро-дифференциальных уравнений с ядрами, имеющими интегрируемые особенности, посвящены работы [6-9], в которых изучается сильная разрешимость указанных уравнений, а также проводится их спектральный анализ.

Теорема 2. Предположим, что вектор-функиия $f(t) \in L_{2, \gamma_{0}}\left(\mathbb{R}_{+}, H\right)$ для некоторого $\gamma_{0}>0$, ядро $K(t)$ представимо в виде (9), а также выполняется условие (10), кроме того, $\varphi_{0} \in H_{2}$, $\varphi_{1} \in H$. Тогда существует такое $\gamma_{1}>\gamma_{0}$, что для всех $\gamma \geqslant \gamma_{1}$ задача (6)-(8) имеет обобщенное решение в пространстве $W_{2, \gamma}^{1}\left(\mathbb{R}_{+}, A\right)$, удовлетворяющее неравенству

$$
\|u\|_{W_{2, \gamma}^{2}\left(\mathbb{R}_{+}, A^{2}\right)} \leqslant d\left(\|f\|_{L_{2, \gamma}\left(\mathbb{R}_{+}, H\right)}+\left\|A^{2} \varphi_{0}\right\|_{H}+\left\|A \varphi_{1}\right\|_{H}\right),
$$

c постоянной $d$, не зависящей от вектор-функции $f$ и векторов $\varphi_{0}, \varphi_{1}$.

3. Доказательства теорем 1 и 2. Доказательству теорем 1 и 2 предпошлем следующие предложения.

Предложение 1. Для любого $\gamma \geqslant \gamma_{1}>0$ и для всех $n \in \mathbb{N}$ существуют такие положительные константы $d_{1} u d_{2}$, что в полуплоскости $\{\lambda: \operatorname{Re} \lambda>\gamma\}$ справедливы неравенства

$$
\begin{aligned}
& \sup _{\operatorname{Re} \lambda>\gamma}\left|\frac{a_{n}}{l_{n}(\lambda)}\right| \leqslant d_{1}<\infty, \\
& \sup _{\operatorname{Re} \lambda>\gamma}\left|\frac{\lambda}{l_{n}(\lambda)}\right| \leqslant d_{2}<\infty .
\end{aligned}
$$

Доказательство. Пусть $\lambda=x+i y=|\lambda|(\cos \varphi+i \sin \varphi)$. Рассмотрим функцию

$$
\mathfrak{M}_{n}(\lambda)=\frac{l_{n}(\lambda)}{a_{n}^{2}}
$$


и оценим снизу $\left|\mathfrak{M}_{n}(\lambda)\right|$. Вычисляя вещественную и мнимую части функции $\mathfrak{M}_{n}(\lambda)$, получим

$$
\begin{aligned}
& \operatorname{Re} \mathfrak{M}_{n}(\lambda)=\frac{x^{2}-y^{2}}{a_{n}^{2}}+1-\int_{0}^{\infty} \frac{(x+\tau) d \mu(\tau)}{(x+\tau)^{2}+y^{2}}, \quad \lambda=x+i y, \\
& \operatorname{Im} \mathfrak{M}_{n}(\lambda)=\frac{2 x y}{a_{n}^{2}}+y \int_{0}^{\infty} \frac{d \mu(\tau)}{(x+\tau)^{2}+y^{2}}, \quad n \in \mathbb{N} .
\end{aligned}
$$

Разделим полуплоскость $\left\{\lambda: \operatorname{Re} \lambda>\gamma_{0}\right\}$ на две области

$$
\Omega_{1}=\left\{|y|>\operatorname{Re} \lambda:=x>\gamma_{0}, y=\operatorname{Im} \lambda\right\}, \quad \Omega_{2}=\{\lambda: \operatorname{Re} \lambda=x>|y|, y=\operatorname{Im} \lambda\} .
$$

Вначале проведем оценку величины $\left|a_{n} / l_{n}(\lambda)\right|$ в области $\Omega_{1}$. Имеем

$$
\begin{array}{r}
\frac{\left|l_{n}(\lambda)\right|}{a_{n}^{2}} \geqslant\left|\operatorname{Im} \mathfrak{M}_{n}(\lambda)\right|>|y|\left(\frac{2 \gamma}{a_{n}^{2}}+\frac{1}{y^{2}} \int_{d_{1}}^{\infty} \frac{d \mu(\tau)}{1+(x+\tau)^{2} / y^{2}}\right)>\frac{2 \gamma|y|}{a_{n}^{2}}+\frac{1}{|y|} \int_{d_{1}}^{\infty} \frac{d \mu(\tau)}{1+(1+\tau /|y|)^{2}}> \\
>\frac{2 \gamma|y|}{a_{n}^{2}}+\frac{\eta_{0}(\gamma)}{|y|}
\end{array}
$$

где

$$
\eta_{0}(\gamma)=\int_{d_{1}}^{\infty} \frac{d \mu(\tau)}{1+(1+\tau / \gamma)^{2}}
$$

и $0<d_{1}<+\infty$. Отсюда получаем

$$
\left|\operatorname{Im} \mathfrak{M}_{n}(\lambda)\right|>\frac{2 \gamma y^{2}+\eta_{0}(\gamma) a_{n}^{2}}{a_{n}^{2}|y|} \geqslant \frac{\sqrt{2 \gamma \eta_{0}}}{a_{n}}=\frac{k(\gamma)}{a_{n}} .
$$

Из последнего неравенства при $|y|>x>\gamma>0$ получаем оценку

$$
\left|\frac{a_{n}}{l_{n}(\lambda)}\right| \leqslant \frac{1}{\sqrt{2 \gamma \cdot \eta_{0}(\gamma)}}=\frac{1}{k(\gamma)}
$$

где $k(\gamma)$ - положительная константа, зависящая от параметра $\gamma>0$. Следовательно, для всех $\lambda \in \Omega_{1}$ получаем оценку

$$
\frac{a_{n}}{\left|l_{n}(\lambda)\right|} \leqslant \frac{1}{k(\gamma)}
$$

Проведем теперь оценку величины $\left|a_{n} / l_{n}(\lambda)\right|$ в области $\Omega_{2}$. Заметим, что при выполнении условия (10) справедлива следующая цепочка неравенств:

$$
\int_{0}^{\infty} \frac{(x+\tau) d \mu(\tau)}{(x+\tau)^{2}+y^{2}} \leqslant \int_{0}^{\infty} \frac{d \mu(\tau)}{(x+\tau)} \leqslant \int_{0}^{\infty} \frac{d \mu(\tau)}{\gamma+\tau}<\int_{0}^{\infty} \frac{d \mu(\tau)}{\tau}<1 .
$$

Таким образом, при $x>|y|$ справедлива оценка

$$
\frac{\left|l_{n}(\lambda)\right|}{a_{n}^{2}} \geqslant \operatorname{Re} \mathfrak{M}_{n}(\lambda) \geqslant \frac{x^{2}-y^{2}}{a_{n}^{2}}+1-\int_{0}^{\infty} \frac{d \mu(\tau)}{\tau+\gamma} \geqslant 1-\int_{0}^{\infty} \frac{d \mu(\tau)}{\tau+\gamma}=: \theta_{0}>0 .
$$

Следовательно, для всех $\lambda \in \Omega_{2}$ получаем оценку

$$
\frac{a_{n}}{\left|l_{n}(\lambda)\right|}<\frac{1}{a_{n}\left|\operatorname{Re} \mathfrak{M}_{n}(\lambda)\right|}<\frac{1}{a_{n} \theta_{0}} .
$$

Объединяя неравенства (21) и (22), приходим к тому, что в полуплоскости $\{\lambda: \operatorname{Re} \lambda=x>\gamma\}$ выполнена оценка

$$
\sup _{\substack{\operatorname{Re} \lambda>\gamma \\ n \in \mathbb{N}}} \frac{a_{n}}{\left|l_{n}(\lambda)\right|} \leqslant \max \left\{\frac{1}{\sqrt{2 \gamma \eta_{0}}} ; \frac{1}{a_{1} \theta_{0}}\right\}=: d_{1} .
$$


Неравенство (19) установлено.

Оценим для всех $\lambda=x+i y, x>\gamma$ выражение $\left|\lambda / l_{n}(\lambda)\right|$. Имеем

$$
\begin{aligned}
&\left|\frac{l_{n}(\lambda)}{\lambda}\right|=\left|\lambda+\frac{a_{n}^{2}}{\lambda}\left(1-\int_{0}^{\infty} \frac{d \mu(\tau)}{\lambda+\tau}\right)\right| \geqslant \\
& \qquad\left|\operatorname{Re} \frac{l_{n}(\lambda)}{\lambda}\right| \geqslant\left|x+\frac{a_{n}^{2} x}{x^{2}+y^{2}}\left(1-\int_{0}^{\infty} \frac{(x+\tau) d \mu(\tau)}{\left((x+\tau)^{2}+y^{2}\right)}\right)\right|>\gamma .
\end{aligned}
$$

Используя оценку (24), при $x>\gamma>\gamma_{1} \geqslant 0$ получаем

Следовательно,

$$
\left|\frac{\lambda}{l_{n}(\lambda)}\right|<\left|\operatorname{Re} \frac{l_{n}(\lambda)}{\lambda}\right|^{-1}<\frac{1}{\gamma} \text {. }
$$

$$
\sup _{\substack{\operatorname{Re} \lambda>\gamma \\ n \in \mathbb{N}}}\left|\frac{1}{l_{n}(\lambda)}\right|<\frac{1}{\gamma}=: d_{2}
$$

Таким образом, получаем искомую оценку (20). Предложение 1 доказано.

В свою очередь из неравенств (19), (20) имеем

$$
\sup _{\operatorname{Re} \lambda>\gamma}\left\|A L^{-1}(\lambda)\right\| \leqslant d_{1}<\infty, \sup _{\operatorname{Re} \lambda>\gamma}\left\|\lambda L^{-1}(\lambda)\right\| \leqslant d_{2}<\infty .
$$

Положим

$$
h(t)=\int_{0}^{t} K(t-s) A^{2}\left(\cos (A s) \varphi_{0}+A^{-1} \sin (A s) \varphi_{1}\right) d s .
$$

Предложение 2. Пусть выполнены условия теоремы 1 ; тогда для любого $\gamma \geqslant \gamma_{1}>\gamma_{0}$ справедлива оценка

$$
\|h(t)\|_{L_{2, \gamma}\left(\mathbb{R}_{+}, H\right)} \leqslant d_{3}\left(\left\|A^{2} \varphi_{0}\right\|+\left\|A \varphi_{1}\right\|\right)
$$

с постоянной $d_{3}$, не зависящей от $\varphi_{0} u \varphi_{1}$.

Доказательство. Оценим вектор-функцию $h(t)$. С этой целью оценим вектор-функцию $\hat{h}(\lambda)$ в пространстве Харди $H_{2}(\operatorname{Re} \lambda>\gamma, H)$. Вектор-функция $\hat{h}(\lambda)$ допускает представление

Следовательно,

$$
\hat{h}(\lambda)=\hat{K}(\lambda)\left[\lambda\left(\lambda^{2} I+A^{2}\right)^{-1} A^{2} \varphi_{0}+A\left(\lambda^{2} I+A^{2}\right)^{-1} A \varphi_{1}\right] .
$$

$$
\begin{aligned}
& \|\hat{h}(\lambda)\|_{H_{2}(\operatorname{Re} \lambda>\gamma, H)}^{2}= \\
& \quad=\sup _{x>\gamma} \int_{-\infty}^{+\infty}\left\|\hat{K}(x+i y)\left[(x+i y)\left((x+i y)^{2} I+A^{2}\right)^{-1} A^{2} \varphi_{0}+A\left((x+i y)^{2} I+A^{2}\right)^{-1} A \varphi_{1}\right]\right\|_{H}^{2} d y .
\end{aligned}
$$

Проведем оценку полученного интеграла.

$$
\begin{aligned}
& \left\|\hat{K}(x+i y)\left[(x+i y)\left((x+i y)^{2} I+A^{2}\right)^{-1} A^{2} \varphi_{0}+A\left((x+i y)^{2} I+A^{2}\right)^{-1} A \varphi_{1}\right]\right\|_{H}^{2} \leqslant \\
& \leqslant C\left|\int_{0}^{\infty} \frac{d \mu(\tau)}{x+i y+\tau}\right|^{2}\left(\left\|(x+i y)\left((x+i y)^{2} I+A^{2}\right)^{-1} A^{2} \varphi_{0}\right\|_{H}^{2}++\left\|A\left((x+i y)^{2} I+A^{2}\right)^{-1} A \varphi_{1}\right\|_{H}^{2}\right) \leqslant \\
& \leqslant C\left(\int_{0}^{\infty} \frac{d \mu(\tau)}{|x+i y+\tau|}\right)^{2}\left(\sum_{n=1}^{\infty} \frac{\left(x^{2}+y^{2}\right) a_{n}^{4}\left|\varphi_{0 n}\right|^{2}}{\left(\left(x^{2}-y^{2}+a_{n}^{2}\right)^{2}+4 x^{2} y^{2}\right)}+\sum_{n=1}^{\infty} \frac{a_{n}^{4}\left|\varphi_{1 n}\right|^{2}}{\left(\left(x^{2}-y^{2}+a_{n}^{2}\right)^{2}+4 x^{2} y^{2}\right)}\right) .
\end{aligned}
$$


Заметим, что

$$
\left(x^{2}-y^{2}+a_{n}^{2}\right)^{2}+4 x^{2} y^{2}=\left(x^{2}+\left(y-a_{n}^{2}\right)\right)\left(x^{2}+\left(y+a_{n}\right)^{2}\right) .
$$

Кроме того, при $x>\gamma$ справедливо неравенство

$$
\int_{0}^{\infty} \frac{d \mu(\tau)}{|x+i y+\tau|} \leqslant \int_{0}^{\infty} \frac{d \mu(\tau)}{x+\tau} \leqslant \int_{0}^{\infty} \frac{d \mu(\tau)}{\tau}<1 .
$$

Используя оценку (28), получаем оценку для интеграла

$$
\begin{aligned}
& \|\hat{h}(\lambda)\|_{H_{2}(\operatorname{Re} \lambda>\gamma, H)}^{2} \leqslant \\
& \leqslant C \sup _{x>\gamma} \sum_{n=1}^{\infty}\left(\int_{-\infty}^{+\infty} \frac{\left(x^{2}+y^{2}\right) a_{n}^{4}\left|\varphi_{0 n}\right|^{2}}{\left(x^{2}+\left(y-a_{n}^{2}\right)\left(x^{2}+\left(y+a_{n}\right)^{2}\right)\right.} d y+\int_{-\infty}^{+\infty} \frac{a_{n}^{4}\left|\varphi_{1 n}\right|^{2}}{\left(x^{2}+\left(y-a_{n}^{2}\right)\left(x^{2}+\left(y+a_{n}\right)^{2}\right)\right.} d y\right) \leqslant \\
& \leqslant 2 C \sup _{x>\gamma} \sum_{n=1}^{\infty}\left(\int_{0}^{+\infty} \frac{a_{n}^{4}\left|\varphi_{0 n}\right|^{2}}{\left(x^{2}+\left(y-a_{n}^{2}\right)\right)} d y+\int_{0}^{+\infty} \frac{a_{n}^{4}\left|\varphi_{1 n}\right|^{2}}{a_{n}^{2}\left(x^{2}+\left(y-a_{n}^{2}\right)\right)} d y\right) \leqslant \\
& \leqslant C \frac{2 \pi}{\gamma}\left(\sum_{n=1}^{\infty} a_{n}^{4}\left|\varphi_{0 n}\right|^{2}+\sum_{n=1}^{\infty} a_{n}^{2}\left|\varphi_{1 n}\right|^{2}\right)=C \frac{2 \pi}{\gamma}\left(\left\|A^{2} \varphi_{0}\right\|_{H}^{2}+\left\|A \varphi_{1}\right\|_{H}^{2}\right) .
\end{aligned}
$$

3.1. Доказательство теоремъ 1. Начнем с доказательства теоремы 1 в случае однородных (нулевых) начальных условий $\varphi_{0}=\varphi_{1}=0$. При доказательстве теоремы 1 с нулевыми начальными условиями используется схема доказательства корректной разрешимости задачи Коши для уравнений гиперболического типа, основанная на применении преобразования Лапласа. В связи с этим, для удобства читателя, напомним широко известные факты, которые мы будем использовать в дальнейшем.

Определение 3. Назовем пространством Харди $H_{2}(\operatorname{Re} \lambda>\gamma, H)$ класс вектор-функций $\hat{f}(\lambda)$ со значениями в $H$, голоморфных в полуплоскости $\{\lambda \in \mathbb{C}: \operatorname{Re} \lambda>\gamma \geqslant 0\}$, для которых

$$
\sup _{x>\gamma} \int_{-\infty}^{+\infty}\|\hat{f}(x+i y)\|_{H}^{2} d y<\infty, \quad \lambda=x+i y .
$$

Сформулируем теорему Пэли-Винера для пространств Харди $H_{2}(\operatorname{Re} \lambda>\gamma, H)$.

Теорема 3. Имеют место следующие утверждения.

1. Пространство $\mathrm{H}_{2}(\operatorname{Re} \lambda>\gamma, H)$ совпадает с множеством вектор-функций (преобразований Лапласа), допускающих представление

$$
\hat{f}(\lambda)=\frac{1}{\sqrt{2 \pi}} \int_{0}^{\infty} e^{-\lambda t} f(t) d t
$$

где $f(t) \in L_{2, \gamma}\left(\mathbb{R}_{+}, H\right), \lambda \in \mathbb{C}, \operatorname{Re} \lambda>\gamma \geqslant 0$.

2. Для любой вектор-функиии $\hat{f}(\lambda) \in H_{2}(\operatorname{Re} \lambda>\gamma, H)$ существует и единственно представление (30), где вектор-функиия $f(t) \in L_{2, \gamma}\left(\mathbb{R}_{+}, H\right)$, причем справедлива формула обращения

$$
f(t)=\frac{1}{\sqrt{2 \pi}} \int_{-\infty}^{+\infty} \hat{f}(\gamma+i y) e^{(\gamma+i y) t} d y, \quad t \in \mathbb{R}_{+}, \quad \gamma \geqslant 0 .
$$


3. Для вектор функиий $\hat{f}(\lambda) \in H_{2}(\operatorname{Re} \lambda>\gamma, H) u f(t) \in L_{2, \gamma}\left(\mathbb{R}_{+}, H\right)$, связанных соотношением (30), справедливо равенство

$$
\|\hat{f}\|_{H_{2}(\operatorname{Re} \lambda>\gamma, H)}^{2} \equiv \sup _{x>\gamma} \int_{-\infty}^{+\infty}\|\hat{f}(x+i y)\|_{H}^{2} d y=\int_{0}^{+\infty} e^{-2 \gamma t}\|f(t)\|_{H}^{2} d t \equiv\|f\|_{L_{2, \gamma}\left(\mathbb{R}_{+}, H\right)}^{2} .
$$

Сформулированная теорема широко известна для скалярных функций. Однако без труда обобщается на случай вектор-функций со значениями в сепарабельном гильбертовом пространстве.

Вернемся к доказательству теоремы 1. Вначале рассмотрим задачу (6), (7) с нулевыми начальными данными $\varphi_{0}=\varphi_{1}=0$. Применяя преобразование Лапласа к уравнению (6), получаем следующее представление для преобразования Лапласа решения задачи (6), (7):

$$
\hat{u}(\lambda)=L^{-1}(\lambda) \hat{f}(\lambda) .
$$

Перейдем к доказательству однозначной разрешимости задачи (6), (7) в пространстве $W_{2, \gamma}^{2}\left(\mathbb{R}_{+}, A^{2}\right)$. Покажем вначале, что вектор-функция $A^{2} u(t) \in L_{2, \gamma}\left(\mathbb{R}_{+}, H\right)$. Легко видеть, что

$$
A^{2} \hat{u}(\lambda)=A^{2} L^{-1}(\lambda) \hat{f}(\lambda)=A L^{-1}(\lambda) A \hat{f}(\lambda) .
$$

Согласно теореме Пэли-Винера $A \hat{f}(\lambda) \in H_{2}\left(\operatorname{Re} \lambda>\gamma_{0} ; H\right)$, поскольку $A f(t) \in L_{2, \gamma_{0}}\left(\mathbb{R}_{+}, H\right)$. Кроме того, справедливо равенство

$$
\|A f\|_{L_{2, \gamma_{0}}\left(\mathbb{R}_{+}, H\right)}=\|A \hat{f}\|_{H_{2}\left(\operatorname{Re} \lambda>\gamma_{0}, H\right)} .
$$

Согласно (26), (34), (35) получаем цепочку неравенств

$$
\left\|A^{2} u\right\|_{L_{2, \gamma}\left(\mathbb{R}_{+}, H\right)}^{2}=\left\|A^{2} \hat{u}\right\|_{H_{2}(\operatorname{Re} \lambda>\gamma, H)}^{2}=\left\|A L^{-1}(\lambda) A \hat{f}(\lambda)\right\|_{H_{2}(\operatorname{Re} \lambda>\gamma, H)}^{2} \leqslant d_{1}^{2}\|A f\|_{L_{2, \gamma}\left(\mathbb{R}_{+}, H\right)}^{2} .
$$

Таким образом, вектор-функция $A^{2} u(t)$ принадлежит пространству $L_{2, \gamma}\left(\mathbb{R}_{+}, H\right)$, и справедлива следующая оценка

$$
\left\|A^{2} u\right\|_{L_{2, \gamma}\left(\mathbb{R}_{+}, H\right)} \leqslant d_{1}\|A f\|_{L_{2, \gamma}\left(\mathbb{R}_{+}, H\right)} .
$$

Покажем, что вектор-функция $\lambda^{2} \hat{u}(\lambda)$ также принадлежит пространству $H_{2}(\operatorname{Re} \lambda>\gamma, H)$. Заметим, что при $\operatorname{Re} \lambda>\gamma$ справедливо представление

$$
I=\lambda^{2} L^{-1}(\lambda)+(1-\hat{K}(\lambda)) A^{2} L^{-1}(\lambda) .
$$

Следовательно, при $\operatorname{Re} \lambda>\gamma$ имеем

$$
\hat{f}(\lambda)=\lambda^{2} \hat{u}(\lambda)+(1-\hat{K}(\lambda)) A^{2} L^{-1}(\lambda) \hat{f}(\lambda) .
$$

В силу предположений относительно функции $K(t)$ функция $1-\hat{K}(\lambda)$ является ограниченной и аналитической в полуплоскости $\{\lambda: \operatorname{Re} \lambda=x>\gamma\}$. В самом деле, справедливо следующее неравенство:

$$
|1-\hat{K}(\lambda)| \leqslant 1+\int_{0}^{\infty} \frac{d \mu(\tau)}{|\lambda+\tau|} \leqslant 1+\int_{0}^{\infty} \frac{d \mu(\tau)}{x+\tau} \leqslant 1+\int_{0}^{\infty} \frac{d \mu(\tau)}{\tau}<2 .
$$

Оценим вектор-функцию $\lambda^{2} \hat{u}(\lambda)$ в пространстве Харди $H_{2}(\operatorname{Re} \lambda>\gamma, H)$. Из представления (39) неравенства (26) и предыдущей оценки получаем

$$
\begin{array}{r}
\left\|\lambda^{2} \hat{u}(\lambda)\right\|_{H_{2}(\operatorname{Re} \lambda>\gamma, H)} \leqslant\|\hat{f}(\lambda)\|_{H_{2}(\operatorname{Re} \lambda>\gamma, H)}+|1-\hat{K}(\lambda)| \cdot\left\|A L^{-1}(\lambda) A f(\lambda)\right\|_{H_{2}(\operatorname{Re} \lambda>\gamma, H)} \leqslant \\
\leqslant \operatorname{const}\|A f(\lambda)\|_{H_{2}(\operatorname{Re} \lambda>\gamma, H)} .
\end{array}
$$

Таким образом, из теоремы Пэли-Винера вытекает неравенство

$$
\left\|\frac{d^{2} u}{d t^{2}}\right\|_{L_{2, \gamma}\left(\mathbb{R}_{+}, H\right)}^{2} \leqslant d_{1}\|A f\|_{L_{2, \gamma}\left(\mathbb{R}_{+}, H\right)}^{2} .
$$


Наконец, объединяя оценки (37) и (41), мы получаем, что вектор-функция $u(t)$ принадлежит пространству $W_{2, \gamma}^{2}\left(\mathbb{R}_{+}, H\right)$ и справедлива оценка

$$
\|u\|_{W_{2, \gamma}^{2}\left(\mathbb{R}_{+}, A^{2}\right)} \leqslant d_{2}\|A f\|_{L_{2, \gamma}\left(\mathbb{R}_{+}, H\right)} .
$$

Рассмотрим теперь задачу (6), (7) с неоднородными начальными данными $\varphi_{0}$ и $\varphi_{1}$. Положим

$$
u(t)=\cos (A t) \varphi_{0}+A^{-1} \sin (A t) \varphi_{1}+w(t) .
$$

Тогда вектор-функция $w(t)$ является решением следующей задачи

$$
\frac{d^{2} w}{d t^{2}}+A^{2} w(t)-\int_{0}^{t} K(t-s) A^{2} w(s) d s=f_{1}(t), \quad w(+0)=w^{(1)}(+0)=0,
$$

где $f_{1}(t)=f(t)-h(t)$,

$$
h(t)=\int_{0}^{t} K(t-s) A^{2}\left(\cos (A s) \varphi_{0}+A^{-1} \sin (A s) \varphi_{1}\right) d s .
$$

Для доказательства теоремы достаточно установить следующее неравенство:

$$
\left\|A f_{1}\right\|_{L_{2, \gamma}\left(\mathbb{R}_{+}, H\right)} \leqslant\|A f\|_{L_{2, \gamma}\left(\mathbb{R}_{+}, H\right)}+\|A h\|_{L_{2, \gamma}\left(\mathbb{R}_{+}, H\right)}<\infty .
$$

Из оценки (27) вытекает требуемая для доказательства теоремы 1 оценка

$$
\|A h(t)\|_{L_{2, \gamma}\left(\mathbb{R}_{+}, H\right)} \leqslant d_{4}\left(\left\|A^{3} \varphi_{0}\right\|+\left\|A^{2} \varphi_{1}\right\|\right)
$$

с постоянной $d_{4}$, не зависящей от $\varphi_{0}$ и $\varphi_{1}$.

Докажем единственность сильного решения задачи (6), (7). Предположим, что существуют два различных сильных решения задачи $(6),(7) u_{1}(t)$ и $u_{2}(t)$. Тогда вектор функция $v(t)=u_{1}(t)-$ $u_{2}(t)$ является сильным решением задачи $(6),(7)$ с нулевой правой частью $f(t) \equiv 0$ и нулевыми начальными векторами $\varphi_{0}=\varphi_{1}=0$, и его преобразование Лапласа $\hat{v}(\lambda)$ удовлетворяет уравнению $L(\lambda) \hat{v}(\lambda)=0$. Следовательно, $\hat{v}(\lambda)=0$ и в соответствии с формулой обращения преобразования Лапласа $v(t) \equiv 0$. Теорема 1 доказана.

3.2. Доказательство теоремы 2. Пусть $u(t)$ - сильное решение задачи (6)-(8) с нулевыми начальными данными $\varphi_{0}=\varphi_{1}=0$. Применяя преобразование Лапласа к уравнению (6), получаем следующее представление для преобразования Лапласа сильного решения $u(t)$ задачи (6)-(8):

$$
\hat{u}(\lambda)=L^{-1}(\lambda) \hat{f}(\lambda) .
$$

Рассмотрим проекцию $u_{n}(t)$ вектор-функции $u(t)$ на одномерное подпространство, натянутое на вектор $e_{n}$, т.е. $u_{n}(t)=\left(u(t), e_{n}\right)_{H}$, тогда $\hat{u}_{n}(\lambda)=\left(\hat{u}(\lambda), e_{n}\right)_{H}=l_{n}^{-1}(\lambda) \hat{f}_{n}(\lambda)$.

Перейдем к доказательству обобщенной разрешимости задачи $(6),(7)$ с нулевыми начальными данными $\varphi_{0}=\varphi_{1}=0$ в пространстве $W_{2, \gamma}^{1}\left(\mathbb{R}_{+}, A\right)$ для любого $\gamma \geqslant \gamma_{1}>\gamma_{0}$.

Утверждение 1. Если функиия $u_{n}(t) e_{n}$ является сильным решением задачи

$$
\frac{d^{2} u}{d t^{2}}+A^{2} u-\int_{0}^{t} K(t-s) A^{2} u(s) d s=f_{n}(t) e_{n}, \quad t \in \mathbb{R}_{+}, \quad u(+0)=0, \quad u^{(1)}(+0)=0,
$$

то функиия $u_{n}(t) e_{n}$ является обобщенным решением этой задачи.

Действительно, умножим скалярно уравнение (47) на функцию $v(t) \in W_{2, \gamma}^{1}\left(\mathbb{R}_{+}, A\right)$ в пространстве $L_{2, \gamma}\left(\mathbb{R}_{+}, H\right)$, где $\gamma \geqslant \gamma_{1}>\gamma_{0}$ : 


$$
\begin{array}{r}
\int_{0}^{+\infty}\left(u_{n}^{\prime \prime}(t) e_{n}, v(t)\right)_{H} e^{-2 \gamma t} d t+\int_{0}^{+\infty}\left(A^{2}\left[u_{n}(t) e_{n}-\int_{0}^{t} K(t-s) u_{n}(s) e_{n} d s\right], v(t)\right) e_{H} e^{-2 \gamma t} d t= \\
=\int_{0}^{+\infty}\left(f_{n}(t) e_{n}, v(t)\right)_{H} e^{-2 \gamma t} d t .
\end{array}
$$

Интегрируя по частям первое слагаемое, получаем следующее равенство:

$$
\int_{0}^{+\infty}\left(u_{n}^{\prime \prime}(t) e_{n}, v(t)\right)_{H} e^{-2 \gamma t} d t=-\left(\varphi_{1}, v(0)\right)_{H}-\left\langle u_{n}^{\prime}(t), v^{\prime}(t)\right\rangle_{L_{2, \gamma}\left(\mathbb{R}_{+}, H\right)}+2 \gamma\left\langle u_{n}^{\prime}(t), v(t)\right\rangle_{L_{2, \gamma}\left(\mathbb{R}_{+}, H\right)} .
$$

Преобразуя второе слагаемое, имеем

$$
\begin{gathered}
\int_{0}^{+\infty}\left(A^{2}\left[u_{n}(t) e_{n}-\int_{0}^{t} K(t-s) u_{n}(s) e_{n} d s\right], v(t)\right)_{H} e^{-2 \gamma t} d t= \\
=\left\langle A\left[u_{n}(t) e_{n}-\int_{0}^{t} K(t-s) u_{n}(s) e_{n} d s\right], A v(t)\right\rangle_{L_{2, \gamma}\left(\mathbb{R}_{+}, H\right)} .
\end{gathered}
$$

Таким образом, функция $u_{n}(t) e_{n}$ удовлетворяет тождеству

$$
\begin{aligned}
\left\langle A\left[u_{n}(t) e_{n}+\int_{0}^{t} K(t-s) u_{n}(s) e_{n} d s\right],\right. & A v(t)\rangle_{L_{2, \gamma}\left(\mathbb{R}_{+}, H\right)}-\left\langle u_{n}^{\prime}(t) e_{n}, v^{\prime}(t)\right\rangle_{L_{2, \gamma}\left(\mathbb{R}_{+}, H\right)}+ \\
& +2 \gamma\left\langle u_{n}^{\prime}(t) e_{n}, v(t)\right\rangle_{L_{2, \gamma}\left(\mathbb{R}_{+}, H\right)}=\left\langle f_{n}(t) e_{n}, v(t)\right\rangle_{L_{2, \gamma}\left(\mathbb{R}_{+}, H\right)}
\end{aligned}
$$

и, следовательно, является обобщенным решением задачи (47).

Следствие. Если вектор-функиия

$$
S_{N}(t)=\sum_{n=1}^{N} u_{n}(t) e_{n}
$$

является сильными решениям задачи

$$
\frac{d^{2} u}{d t^{2}}+A^{2} u-\int_{0}^{t} K(t-s) A^{2} u(s) d s=F_{N}(t), \quad t \in \mathbb{R}_{+}, \quad u(+0)=0, \quad u^{(1)}(+0)=0,
$$

где

$$
F_{N}(t)=\sum_{n=1}^{N} f_{n}(t)
$$

то вектор-функиия $S_{N}(t)$ является обобщенным решением задачи (48), т.е. справедливо тождество

$$
\begin{aligned}
\left\langle A\left[S_{N}(t)+\int_{0}^{t} K(t-s) S_{N}(s) d s\right], A v(t)\right\rangle_{L_{2, \gamma}\left(\mathbb{R}_{+}, H\right)}-\left\langle S_{N}^{\prime}(t), v^{\prime}(t)\right\rangle_{L_{2, \gamma}\left(\mathbb{R}_{+}\right)}+ \\
+2 \gamma\left\langle S_{N}^{\prime}(t), v(t)\right\rangle_{L_{2, \gamma}\left(\mathbb{R}_{+}, H\right)}=\left\langle F_{N}(t), v(t)\right\rangle_{L_{2, \gamma}\left(\mathbb{R}_{+}, H\right)}
\end{aligned}
$$


Покажем, что при выполнении условий теоремы 1 вектор-функция

$$
u(t)=\sum_{n=1}^{\infty} u_{n}(t) e_{n}
$$

(где для каждого $n \in \mathbb{N}$ функция $u_{n}(t) e_{n}$ является сильным решением соответствующей задачи (47)) является обобщенным решением задачи (6)-(8).

Для этого покажем, что последовательность

$$
S_{N}(t)=\sum_{n=1}^{N} u_{n}(t) e_{n}
$$

является фундаментальной в пространстве $W_{2, \gamma}^{1}\left(\mathbb{R}_{+}, A\right)$, используя оценки $(19),(20)$ из предложения 1.

$$
\begin{aligned}
& \left\|S_{N}(t)-S_{M}(t)\right\|_{W_{2, \gamma}^{1}\left(\mathbb{R}_{+}, A\right)}^{2}=\left\|\sum_{n=M+1}^{N} u_{n}(t) e_{n}\right\|_{W_{2, \gamma}^{1}\left(\mathbb{R}_{+}, A\right)}^{2}= \\
& =\left\|A \sum_{n=M+1}^{N} u_{n}(t) e_{n}\right\|_{L_{2, \gamma}\left(\mathbb{R}_{+}, H\right)}^{2}+\left\|\sum_{n=M+1}^{N} u_{n}^{\prime}(t) e_{n}\right\|_{L_{2, \gamma}\left(\mathbb{R}_{+}, H\right)}^{2}= \\
& =\left\|A \sum_{n=M+1}^{N} \hat{u}_{n}(\lambda) e_{n}\right\|_{H_{2}(\operatorname{Re} \lambda>0, H)}^{2}+\left\|\sum_{n=M+1}^{N} \lambda \hat{u}_{n}(\lambda) e_{n}\right\|_{H_{2}(\operatorname{Re} \lambda>0, H)}^{2}= \\
& =\sup _{x>\gamma} \int_{-\infty}^{+\infty}\left(\sum_{n=M+1}^{N}\left|a_{n} \hat{u}_{n}(x+i y)\right|^{2}+\sum_{n=M+1}^{N}\left|(x+i y) \hat{u}_{n}(x+i y)\right|^{2}\right) d y= \\
& =\sup _{x>\gamma} \int_{-\infty}^{+\infty}\left(\sum_{n=M+1}^{N}\left|\frac{a_{n} \hat{f}_{n}(x+i y)}{l_{n}(x+i y)}\right|^{2}+\sum_{n=M+1}^{N}\left|\frac{(x+i y) \hat{f}_{n}(x+i y)}{l_{n}(x+i y)}\right|^{2}\right) d y \leqslant \\
& \leqslant \int_{-\infty}^{+\infty} \sum_{n=M+1}^{N} \sup _{x>\gamma}\left(\left|\frac{a_{n}}{l_{n}(x+i y)}\right|^{2}+\left|\frac{x+i y}{l_{n}(x+i y)}\right|^{2}\right)\left|\hat{f}_{n}(x+i y)\right|^{2} d y \leqslant \\
& \leqslant d_{5} \sup _{x>\gamma} \int_{-\infty}^{+\infty} \sum_{n=M+1}^{N}\left|\hat{f}_{n}(x+i y)\right|^{2} d y=d_{5}\left\|\sum_{n=M+1}^{N} \hat{f}_{n}(\lambda) e_{n}\right\|_{H_{2}(\operatorname{Re} \lambda>0, H)}= \\
& =d_{5}\left\|\sum_{n=M+1}^{N} f_{n}(t) e_{n}\right\|_{L_{2, \gamma}\left(\mathbb{R}_{+}, H\right)}^{2} .
\end{aligned}
$$

По условию теоремы 1 вектор-функция $f(t)$ принадлежит пространству $L_{2, \gamma}\left(\mathbb{R}_{+}, H\right)$, следовательно, последовательность вектор-функций $S_{N}(t)$ сходится в пространстве $W_{2, \gamma}^{1}\left(\mathbb{R}_{+}, A\right)$ к векторфункции $u(t)$, при $F_{N}(t)$, стремящейся к $F(t)$ в $L_{2, \gamma}\left(\mathbb{R}_{+}, H\right)$. Переходя к пределу при $N \rightarrow+\infty$ в тождестве (49), получаем тождество (16) при $\varphi_{1}=0$, т.е. функция

$$
u(t)=\sum_{n=1}^{\infty} u_{n}(t) e_{n}
$$

является обобщенным решением задачи (6)-(8).

Получим оценку нормы обобщенного решения

$$
u(t)=\sum_{n=1}^{\infty} u_{n}(t) e_{n}
$$


в пространстве $W_{2, \gamma}^{1}\left(\mathbb{R}_{+}, A\right)$. Полагая $M=0$ и $S_{M}(t)=0$ в цепочке неравенств $(50)$, приходим к неравенству

$$
\left\|S_{N}(t)\right\|_{W_{2, \gamma}^{1}\left(\mathbb{R}_{+}, A\right)}^{2} \leqslant d_{5}\left\|\sum_{n=1}^{N} f_{n}(t) e_{n}\right\|_{L_{2, \gamma}\left(\mathbb{R}_{+}, H\right)}^{2} \leqslant d_{5}\|f(t)\|_{L_{2, \gamma}\left(\mathbb{R}_{+}, H\right)}^{2} .
$$

Переходя к пределу при $N \rightarrow+\infty$ в последнем неравенстве, получаем следующую оценку

$$
\|u(t)\|_{W_{2, \gamma}^{1}\left(\mathbb{R}_{+}, A\right)}^{2} \leqslant d_{5}\|f(t)\|_{L_{2, \gamma}\left(\mathbb{R}_{+}, H\right)}^{2} .
$$

Рассмотрим теперь задачу (6)-(8) с неоднородными начальными данными $\varphi_{0}$ и $\varphi_{1}$. Положим

$$
u(t)=\cos (A t) \varphi_{0}+A^{-1} \sin (A t) \varphi_{1}+w(t) .
$$

Утверждение 2. Вектор-функиия и(t) является обобщенным решением задачи (6)-(8), если вектор-функиия $w(t)$ является обобщенным решением следующей задачи:

$$
\frac{d^{2} w}{d t^{2}}+A^{2} w(t)-\int_{0}^{t} K(t-s) A^{2} w(s) d s=f_{1}(t), \quad w(+0)=w^{(1)}(+0)=0
$$

где $f_{1}(t)=f(t)-h(t)$

$$
h(t)=\int_{0}^{t} K(t-s) A^{2}\left(\cos (A s) \varphi_{0}+A^{-1} \sin (A s) \varphi_{1}\right) d s .
$$

Из условий теоремы 2 и предложения 2 следует справедливость следующей оценки

$$
\left\|f_{1}\right\|_{L_{2, \gamma}\left(\mathbb{R}_{+}, H\right)} \leqslant\|f\|_{L_{2, \gamma}\left(\mathbb{R}_{+}, H\right)}+\|h\|_{L_{2, \gamma}\left(\mathbb{R}_{+}, H\right)}<\infty .
$$

Таким образом, для задачи (53) выполнены условия теоремы 2. Непосредственной подстановкой вектор-функции $u(t)$, заданной формулой (52), в тождество (16) можно убедиться в справедливости утверждения 2. Кроме того, из оценки (51) и предложения 2 следует оценка (18). Теорема 2 доказана.

\section{СПИСОК ЛИТЕРАТУРЫ}

1. Власов В. В., Гавриков А. А., Иванов С. А., Князьков Д. Ю., Самарин В. А., Шамаев А. С. Спектральные свойства комбинированных сред// Совр. пробл. мат. мех. * - 2009. - 5, № 1. - С. 134-155.

2. Власов В. В., Раутиан Н. А. О свойствах решений интегродифференциальных уравнений, возникающих в теории тепломассообмена // Тр. Моск. мат. о-ва. - 2014. - 75, № 2. - С. 131-155.

3. Власов B. В., Раутиан H. А. Корректная разрешимость и спектральный анализ интегродифференциальных уравнений, возникающих в теории вязкоупругости// Совр. мат. Фундам. напр. - 2015. 58. - C. $22-42$.

4. Власов В. В., Раутиан Н. А. Спектральный анализ функционально-дифференциальных уравнений. - M.: MAKC Пресc, 2016.

5. Власов В. В., Раутиан Н. А. Корректная разрешимость вольтерровых интегро-дифференциальных уравнений в гильбертовом пространстве// Диффер. уравн. - 2016. - 52, № 9. - С. 1168-1177.

6. Власов В. В., Раутиан Н. А. Исследование операторных моделей, возникающих в теории вязкоупругости// Совр. мат. Фундам. напр. - 2018. - 64, № 1. - С. 60-73.

7. Власов B. В., Раутиан Н. А. Корректная разрешимость и спектральный анализ вольтерровых интегродифференциальных уравнений с сингулярными ядрами// Докл. РАН. - 2018. - 482, № 6 . C. $635-638$.

8. Власов В. В., Раутиан H. А. Корректная разрешимость и представление решений вольтерровых интегро-дифференциальных уравнений, вонизникающих в теории вязкоупругости// Диффер. уравн. -2019 . - 55, № 4. - C. 574-587.

9. Власов В. В., Раутиан Н. А. Исследование операторных моделей, возникающих в задачах наследственной механики // Тр. семин. им. И. Г. Петровского. - 2019. - 32. - С. 91-110.

10. Жиков В. В. Об одном расширении и применении метода двухмасштабной сходимости// Мат. сб. 2000. -191 , № 7. - C. 31-72. 
11. Илъюшин А. А., Победря Б. Е. Основы математической теории термовязкоупругости. - М.: Наука, 1970.

12. Лыков А. В. Проблема тепло- и массообмена. - Минск: Наука и техника, 1976.

13. Работнов Ю. Н. Элементы наследственной механики твердых тел. - М.: Наука, 1977.

14. Amendola G., Fabrizio M., Golden J. M. Thermodynamics of Materials with Memory. Theory and Applications. - New York-Dordrecht-Heidelberg-London: Springer-Verlag, 2012.

15. Desch W., Miller R. Exponential stabilization of Volterra Integrodifferential equations in Hilbert space// J. Differ. Equations. - 1987. - 70. - P. 366-389.

16. Eremenko A., Ivanov S. Spectra of the Gurtin-Pipkin-type equations// SIAM J. Math. Anal. — 2011. 43, № 5. - P. 2296-2306.

17. Fabrizio M., Gentili G., Golden J. M. Non-isothermal free energies for linear theories with memory// Math. Comp. Model. - 2004. - 39. - P. 219-253.

18. Fabrizio M., Morro A. Mathematical Problems in Linear Viscoelasticity. — Philadelphia, PA: SIAM Stud. Appl. Math., 1992.

19. Gurtin M. E., Pipkin A. C. Theory of heat conduction with finite wave speed// Arch. Rat. Mech. Anal. 1968. - 31. - P. 113-126.

20. Kopachevsky N. D., Krein S. G. Operator Approach to Linear Problems of Hydrodynamics. Vol. 2. Nonself-adjoint Problems for Viscous Fluids. — Basel: Springer-Verlag, 2003.

21. Lions J. L. and Magenes E. Nonhomogeneous Boundary-Value Problems and Applications. - BerlinHeidelberg-New York: Springer-Verlag, 1972.

22. Mahiuddin M., Khan M. I. H., Nghia Duc Pham, Karim M. A. Development of fractional viscoelastic model for characterising viscoelastic properties of food material during drying// Food Biosci. — 2018. — 23. — P. $45-53$.

23. Pandolfi L. The controllability of the Gurtin-Pipkin equations: a cosine operator approach// Appl. Math. Optim. - 2005. - 52. - P. 143-165.

24. Prüss J. Evolutionary Integral Equations amd Applications. - Birkhäuser-Verlag: Basel-Baston-Berlin.

25. Rassoli A., Fatouraee N., Guidoin R. Structural model for viscoelastic properties of pericardial bioprosthetic valves// Artificial Organs. — 2018. - 42. - P. 630-639.

26. Seneta E. Regularly Varying Functions. - Springer-Verlag, 1976.

27. Shahin-Shamsabadi A. et al. Mechanical, material, and biological study of a PCL/bioactive glass bone scaffold: Importance of viscoelasticity// Mat. Sci. Eng. C. - 2018. - 90. - P. 280-288.

28. Stropek Z., Golacki K. Viscoelastic response of apple flesh in a wide range of mechanical loading rates// Int. Agrophys. - 2018. - 32. - P. 335-340.

29. Vlasov V. V., Rautian N. A. Well-defined solvability and spectral analysis of abstract hyperbolic equations// J. Math. Sci. — 2011. — 179, № 3. - P. 390-415.

30. Vlasov $V . V ., W u$ J. Solvability and spectral analysis of abstract hyperbolic equations with delay// Funct. Differ. Equations. - 2009. - 16, № 4. - P. 751-768.

31. $W u$ J. Semigroup and integral form of class of partial differential equations with infinite delay// Differ. Integr. Equations. - 1991. — 4, № 6. - P. 1325-1351.

Раутиан Надежда Александровна

Московский государственный университет им. М. В. Ломоносова

E-mail: nrautian@mail.ru 